\title{
PARASITÓIDES ASSOCIADOS ÀS MOSCAS-DAS-FRUTAS (Diptera: Tephritoidea) EM CAFÉ ORGÂNICO COM E SEM ARBORIZAÇÃO EM VALENÇA, RJ, BRASIL
}

\author{
Parasitoids associated with fruit flies (Diptera: Tephritoidea) in organic coffee plantation \\ under shaded and unshaded management in Valença, RJ, Brazil
}

\author{
Elen de Lima Aguiar-Menezes ${ }^{1}$, Carlos Marcos Alves dos Santos², André Luis Santos Resende \\ Michela Rocha Leal ${ }^{4}$, Euripedes Barsanulfo Menezes ${ }^{5}$
}

\begin{abstract}
RESUMO
Objetivou-se, com esse trabalho, determinar as espécies de parasitóides associados às moscas-das-frutas infestando seis cultivares de café arábica (Coffea arabica L.), em sistema de cultivo com e sem arborização, sob manejo orgânico. Uma amostra de 1 $\mathrm{kg}$ de frutos maduros por cultivar foi colhida em maio de 2005. Os frutos foram depositados em bandejas plásticas, contendo uma camada de areia, a qual serviu como substrato para a fase de pupa. Os pupários foram quantificados e mantidos em copos plásticos transparentes com areia até a emergência dos insetos. Sete espécies de parasitóides da ordem Hymenoptera foram identificadas, das quais cinco da família Braconidae: Asobara anastrephae (Muesebeck), Doryctobracon areolatus (Szépligeti), Utetes anastrephae (Viereck), Opius bellus Gahan e Opius sp., e duas da Figitidae: Aganaspis pelleranoi (Brèthes) e Lopheucoila anastrephae Weld. Larvas de Tephritidae e Lonchaeidae infestando frutos dos seis cultivares foram parasitadas por Braconidae e Figitidae, com média de $14,2 \%$ e $7,7 \%$ nos sistemas sem e com arborização, respectivamente.
\end{abstract}

Termos para indexação: Coffea arabica, Braconidae, Figitidae, parasitismo larval.

\begin{abstract}
The aim of this work was to determine the parasitoid species associated with fruit flies infesting six cultivars of arabic coffee under shaded and unshaded organic management. A $1 \mathrm{~kg}$-sample of maturing fruits per cultivar was harvested in May 2005. The fruits were placed in plastic trays containing a layer of thin sand, as a substrate for pupa phase. The puparia were quantified and kept in transparent plastic cups with sand until the emergence of the insects. Seven parasitoid species of the order Hymenoptera were identified, from which five belong to the family Braconidae: Asobara anastrephae (Muesebeck), Doryctobracon areolatus (Szépligeti), Utetes anastrephae (Viereck), Opius bellus Gahan and Opius sp., and two of the Figitidae: Aganaspis pelleranoi (Brèthes) and Lopheucoila anastrephae Weld. Larvae of Tephritidae and Lonchaeidae infesting fruits of the six cultivars were parasitized by Braconidae and Figitidae, with an average of $14.2 \%$ and $7.7 \%$ in the unshaded and shaded systems, respectively.
\end{abstract}

Index terms: Coffea arabica, Braconidae, Figitidae, larval parasitism.

(Recebido em 9 de janeiro de 2008 e aprovado em 5 de junho de 2008)

\section{INTRODUÇÃO}

O modelo de cafeicultura adotado no Brasil caracteriza-se pelo monocultivo, desconsiderando-se a idéia de que o cafeeiro pode ser cultivado abaixo do dossel das florestas, a exemplo daqueles cultivados na América Central (BEER, 1997). Após a segunda guerra mundial, adotou-se o pacote tecnológico industrial, destacando-se o uso de agroquímicos sintéticos (MATIELLO \&
SIQUEIRA, 1999). Todavia, esse modelo tem se mostrado insustentável do ponto de vista econômico, social e ambiental (HAGGAR et al., 2001).

Novos nichos de mercado para o café vêm crescendo, entre eles, destaca-se o café orgânico. Na cafeicultura orgânica, a diversificação do sistema pode ser obtida pela incorporação de árvores que proporcionam sombra, entre outros benefícios (RICCI et al., 2006), e uma vasta literatura mostra que sistemas agrícolas

\footnotetext{
'Engenheira Agrônoma, PhD, Pesquisadora - Laboratório de Controle Biológico - Embrapa Agrobiologia - Rodovia Br 465, Km 7 - Seropédica, RJ 23890-000 - menezes@cnpab.embrapa.br

2Engenheiro Agrônomo, Mestre em Agricultura Tropical e Subtropical - Instituto Agronômico de Campinas/IAC - Avenida Barão de Itapura, 1481 - Jardim Guanabara - 13020-902 - Campinas, SP - marcosufrrjalves@yahoo.com.br - Bolsista CNPq

3Engenheiro Agrônomo, Mestre - Departamento de Entomologia/DEN - Universidade Federal de Lavras/UFLA - Cx. P. 3037 - $37200-000$ - Lavras, MG alsresende@yahoo.com.br - Bolsista CNPq

${ }^{4}$ Engenheira Agrônoma, Mestre - Departamento de Entomologia e Fitopatologia/DEnF - Universidade Federal Rural do Rio de Janeiro/UFRRJ - Rodovia Br 465, Km 7 - 23890-000 - Seropédica, RJ - michaleal@yahoo.com.br - Bolsista CAPES

${ }^{5}$ Engenheiro Agrônomo, Professor Titular - Departamento de Entomologia e Fitopatologia/DEnF - Universidade Federal Rural do Rio de Janeiro/UFRRJ Rodovia Br 465, Km 7 -23890-000 - Seropédica, RJ - ebmen@uol.com.br
} 
diversificados podem reduzir a incidência de pragas e/ou aumentar a atividade de inimigos naturais (AGUIARMENEZES, 2004).

A infestação por moscas-das-frutas, especialmente as da família Tephritidae, em cafezais tem sido registrada em diferentes regiões do mundo, onde atuam como planta hospedeira de espécies-pragas que danificam frutas de importância econômica (WHITE \& ELSON-HARRIS, 1992). Todavia, essas moscas, especialmente Ceratitis capitata (Wiedemann), são também pragas de café em outros países (ABASA, 1973), e vêm assumindo grande importância econômica em cafezais no Brasil, principalmente na Bahia e Minas Gerais (CIVIDANES et al., 1993; NEVES et al., 2002). Por outro lado, há registros de parasitismo por insetos da ordem Hymenoptera em larvas de moscas-dasfrutas infestando frutos de café no Brasil (GUIMARÃES et al., 1999; LEONEL JUNIOR et al., 1996; RAGA et al., 1996, 2002; TORRES, 2004) e em outros países (ESKAFI, 1990; KATIYAR et al., 1995; WHARTON et al., 1981; YEPES \& VELEZ, 1989), podendo ter seu potencial de uso investigado para o controle biológico dessa praga.

Objetivou-se, neste trabalho, registrar a ocorrência de parasitóides de larvas de moscas-das-frutas (Tephritidae e Lonchaeidae) infestando frutos de seis cultivares de café, sob condições naturais, em manejo orgânico com e sem arborização, no município de Valença, RJ.

\section{MATERIAL E MÉTODOS}

O estudo foi conduzido em áreas experimentais com café arábica (Coffea arabica L.), sob manejo orgânico: uma, com monocultivo de café sem arborização (pleno sol) e a outra com cafeeiros associados a árvores para sombreamento, ambas instaladas a $610 \mathrm{~m}$ de altitude em Barão de Juparanã, Valença, RJ (22¹1'S, 4341'W).

$\mathrm{O}$ delineamento experimental foi o inteiramente casualizado, com quatro repetições, em esquema de parcelas sub-subdivididas. Na parcela avaliaram-se dois sistemas de cultivo: com e sem arborização. Nas subparcelas foram avaliados seis cultivares (Catuaí Vermelho 144, Catucaí Amarelo 2SL, Icatu Amarelo IAC 3282, Obatã IAC 1669-20, Oeiras MG 6851 e Tupi IAC 1669/ 33) e, nas sub-subparcelas, avaliaram-se as espécies de parasitóides identificadas.

Cada parcela experimental media 27,3 m x 10,0 m de largura e era constituída de quatro linhas de cafeeiros, plantados em fevereiro de 2001 no espaçamento de $2,5 \mathrm{~m} \mathrm{x}$ 0,7 m. As duas linhas centrais foram consideradas parcela útil. No sistema arborizado, a bananeira (Musa sp. var. Prata Comum), plantada no espaçamento de $3 \mathrm{~m}$ x $5 \mathrm{~m}$, e a
Erithrina verna Vell., no espaçamento de $9 \mathrm{~m}$ x $5 \mathrm{~m}$, foram utilizadas para sombreamento dos cafeeiros.

Em cada parcela foi coletada aleatoriamente uma amostra de $250 \mathrm{~g}$ de frutos no estágio de cereja distribuídos nos quatro quadrantes das regiões do terço superior e mediano das plantas, totalizando $1 \mathrm{~kg}$ de frutos por cultivar. As amostras foram transportadas em sacos de papel para o laboratório, onde os frutos de cada parcela foram acondicionados separadamente em bandejas plásticas (40 x $30 \times 6 \mathrm{~cm}$ ), contendo areia fina umedecida e autoclavada $( \pm 2 \mathrm{~cm})$ como substrato para a pupação das larvas. Após 15 a 25 dias, o substrato foi peneirado para a coleta dos pupários. Os frutos apodrecidos foram abertos para retirada de pupários remanescentes.

Para cada parcela, os pupários de Tephritidae e Lonchaeidae foram contados e acondicionados separadamente, em copos plásticos transparentes $(250 \mathrm{ml})$, contendo areia fina umedecida e autoclavada $( \pm 2 \mathrm{~cm})$, os quais foram colocados em potes plásticos (2 L) tampados com organza e mantidos a $25 \pm 3^{\circ} \mathrm{C}$ e $80 \%$ UR para emergência dos insetos. Diariamente, por um período de 30 dias, verificou-se a umidade do substrato e observouse a emergência de moscas e de parasitóides. Os insetos foram alimentados com mel e água por três dias, para fixar a coloração que permitisse sua correta identificação, sendo então mortos e conservados em álcool hidratado a $70 \%$, em frascos devidamente rotulados para posterior contagem e identificação específica. Para cada espécie de parasitóide, foi determinada a freqüência relativa como porcentagem de indivíduos de uma espécie em relação ao número total de indivíduos de todas as espécies obtida para cada cultivar e cada sistema de cultivo.

As variáveis determinadas para cada cultivar foi o número de machos e fêmeas de cada espécie de parasitóide identificada e a porcentagem de parasitismo (número de pupas parasitadas dividido pelo número total de pupários e multiplicado por 100). Os pupários sem emergência de adultos foram dissecados para verificação da causa da mortalidade, de modo que o número de adultos de parasitóides não emergidos foi considerado no cálculo da porcentagem de parasitismo. Os tratamentos foram comparados por meio do teste não paramétrico de Kruskal-Wallis $(\mathrm{P}=0,05)$.

\section{RESULTADOS E DISCUSSÃO}

Os espécimes de moscas-das-frutas que infestaram as amostras de café pertencem a duas famílias de Tephritoidea, sendo a maioria da família Tephritidae $(86 \%$ do total de moscas emergidas dos frutos colhidos em cada sistema de cultivo de café). Os $14 \%$ restantes foram espécimes de Lonchaeidae. 
Foram identificadas três espécies de Tephritidae: Ceratitis capitata (Wiedemann), Anastrepha fraterculus (Wiedemann) e Anastrepha sororcula Zucchi (Tabela 1). Essas espécies já foram registradas em café no Brasil (AGUIAR-MENEZES \& MENEZES, 1996; 2000, RAGA et al., 2002; TORRES, 2004), na Costa Rica (WHARTON et al., 1981), Guatemala (ESKAFI \& CUNNINGHAM, 1987), Colômbia (YEPES \& VELEZ, 1989) e Venezuela (KATIYAR et al., 1995).

A maioria das espécies de Lonchaeidae pertencia ao gênero Neosilba McAlpine, totalizando cinco espécies: Neosilba bifida Strikis \& Prado, Neosilba certa (Walker), Neosilba glaberrima (Wiedemann), Neosilba pendula (Bezzi) e Neosilba pseudopendula (Korytkowski and Ojeda), e dois morfotipos: Neosilba sp.1 e Neosilba sp.2 (Tabela 1). Apenas 4\% das moscas Lonchaeidae foram identificadas como Dasiops rugifrons Hennig. N. certa (Walker) foi registrada em café no município de San Pablo, Antioquia, Colômbia (YEPES \& VELEZ, 1989). De acordo com Mcalpine \& Steyskal (1982), as espécies de Lonchaeidae obtidas são invasoras secundárias ou oportunistas por atacarem frutos previamente infestados por larvas de outros insetos, especialmente tefritídeos, com exceção da $N$. bifida e Neosilba sp.1, para as quais ainda não há informações a respeito de seu comportamento alimentar

Os machos de Anastrepha e as fêmeas de Neosilba spp. não foram identificados até o nível de espécie, porque na maioria das espécies desses gêneros, os espécimes desses sexos não apresentam características morfológicas diferenciadoras, sendo as chaves de identificação baseadas nos caracteres morfológicos das fêmeas (ZUCCHI, 2000) e de machos (MCALPINE \& STEYSKAL, 1982), respectivamente. Portanto, para fins de comparação, também só se considerou o número de fêmeas de $C$. capitata e de machos de D. rugifrons (Tabela 1).

Foram identificadas sete espécies de parasitóides, sendo cinco espécies de Braconidae: Asobara anastrephae (Muesebeck), Doryctobracon areolatus (Szépligeti), Utetes anastrephae (Viereck), Opius bellus Gahan e Opius sp., e duas espécies de Figitidae: Aganaspis pelleranoi (Brèthes) e Lopheucoila anastrephae Weld (Tabela 1).

Com exceção de L. anastrephae, as demais espécies já foram registradas como parasitóides de moscas-das-frutas infestando café em outros locais do país (GUIMARÃES et al., 1999; LEONEL JUNIOR et al.,
1996; MALAVASI \& ZUCCHI, 2000; RAGA et al., 1996, 2002; TORRES, 2004). D. areolatus é considerada a espécie mais comum e abundante entre os parasitóides de moscas-das-frutas (WHARTON \& MARSH, 1978). Contudo, em todos os cultivares de café avaliados, $D$. areolatus foi coletada em freqüência inferior a de outras espécies, como $U$. anastrephae e $O$. bellus. É provável que o ovipositor mais curto de $U$. anastrephae e $O$. bellus em relação ao de D. areolatus (GONÇALVES, 1938; SIVINSKI et al., 1999) possa indicar uma especialização daquelas duas espécies em procurar por suas larvas hospedeiras em frutos pequenos, onde podem ser mais hábeis em competir com $D$. areolatus, conforme salientado por López et al. (1999).

Contrariamente aos resultados obtidos, Raga et al. (2002) não observaram a emergência de parasitóides das pupas de tefritídeos obtidas em frutos de Icatu Amarelo, Catucaí Amarelo e Obatã no Estado de São Paulo. A. pelleranoi já foi registrada como parasitóide de C. capitata e A. fraterculus infestando café arábica na Costa Rica (WHARTON et al., 1981) e na Venezuela (KATIYAR et al., 1995). U. anastrephae parasita larvas de A. fraterculus e C. capitata em frutos de café arábica na Colômbia (YEPES \& VELEZ, 1989) e na Guatemala (ESKAFI, 1990), respectivamente, corroborando os resultados obtidos.

A riqueza de espécies de parasitóides obtida no presente estudo foi maior do que a obtida em estudos conduzidos com essa Rubiaceae em outras localidades no país, nas quais se registraram até cinco espécies associadas às moscas-das-frutas em café (GUIMARÃES et al., 1999; MALAVASI \& ZUCCHI, 2000; RAGA et al., 2002; TORRES, 2004). Provavelmente, a riqueza de espécies tenha sido também influenciada pela proximidade das áreas experimentais ao fragmento de Mata Atlântica, na qual as Myrtaceae foram consideradas de alta relevância ecológica por SPOLIDORO (2001) e, por exemplo, A. pelleranoi e $O$. anastrephae buscam suas larvas hospedeiras em diversas espécies frutíferas, porém são mais atraídas por frutos de Myrtaceae (GUIMARÃES et al., 2000). Portanto, espécies frutíferas presentes nesse fragmento devem atuar como repositório natural de multiplicação de parasitóides de moscas-das-frutas, conforme considerado por ALUJA (1999).

Não houve diferença significativa no número de indivíduos das sete espécies de parasitóides identificadas e nas porcentagens de parasitismo total de larvas das moscas-das-frutas entre os seis cultivares de café avaliados 


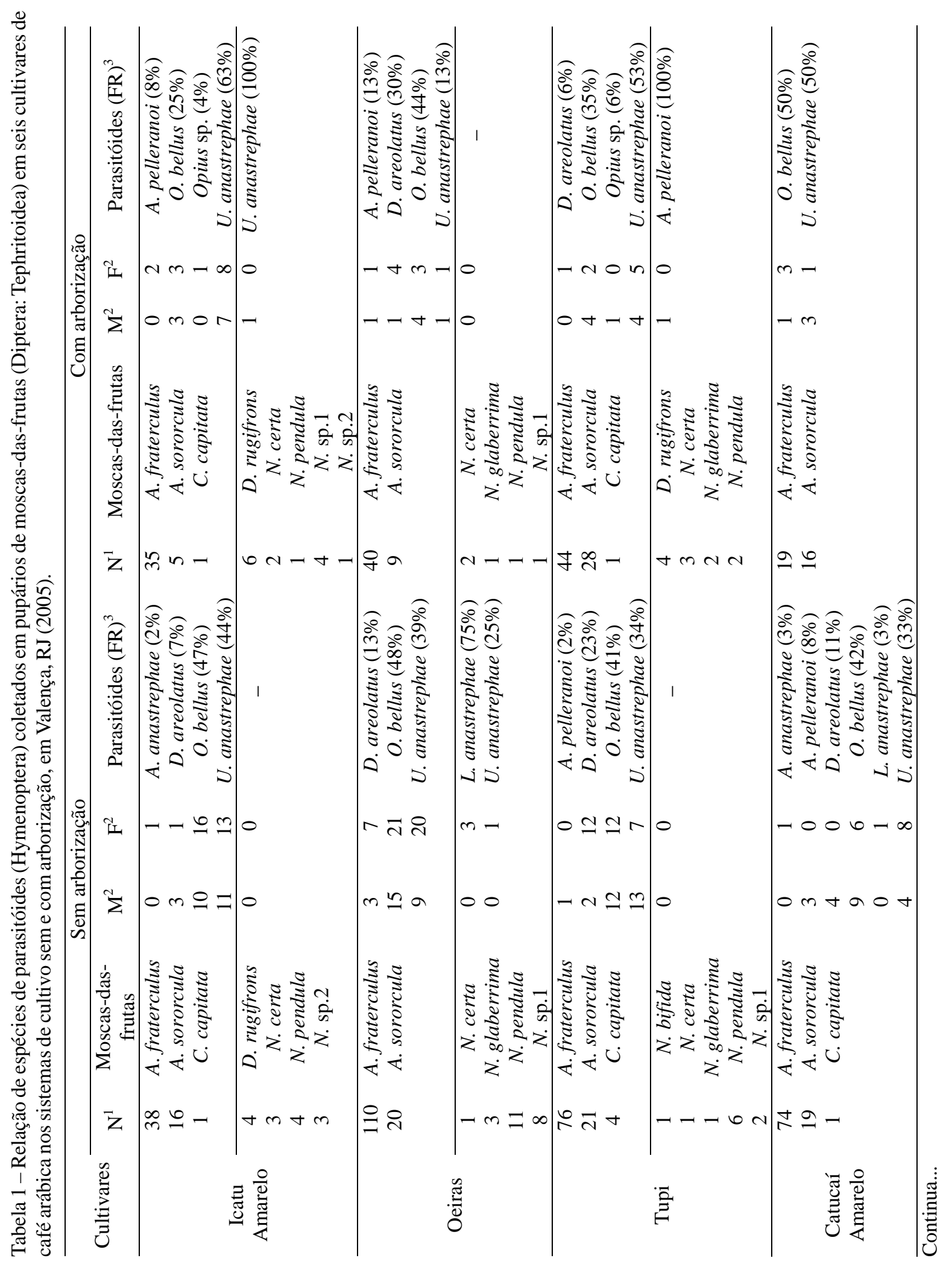

Ciênc. agrotec., Lavras, v. 32, n. 6, p. 1824-1831, nov./dez., 2008 


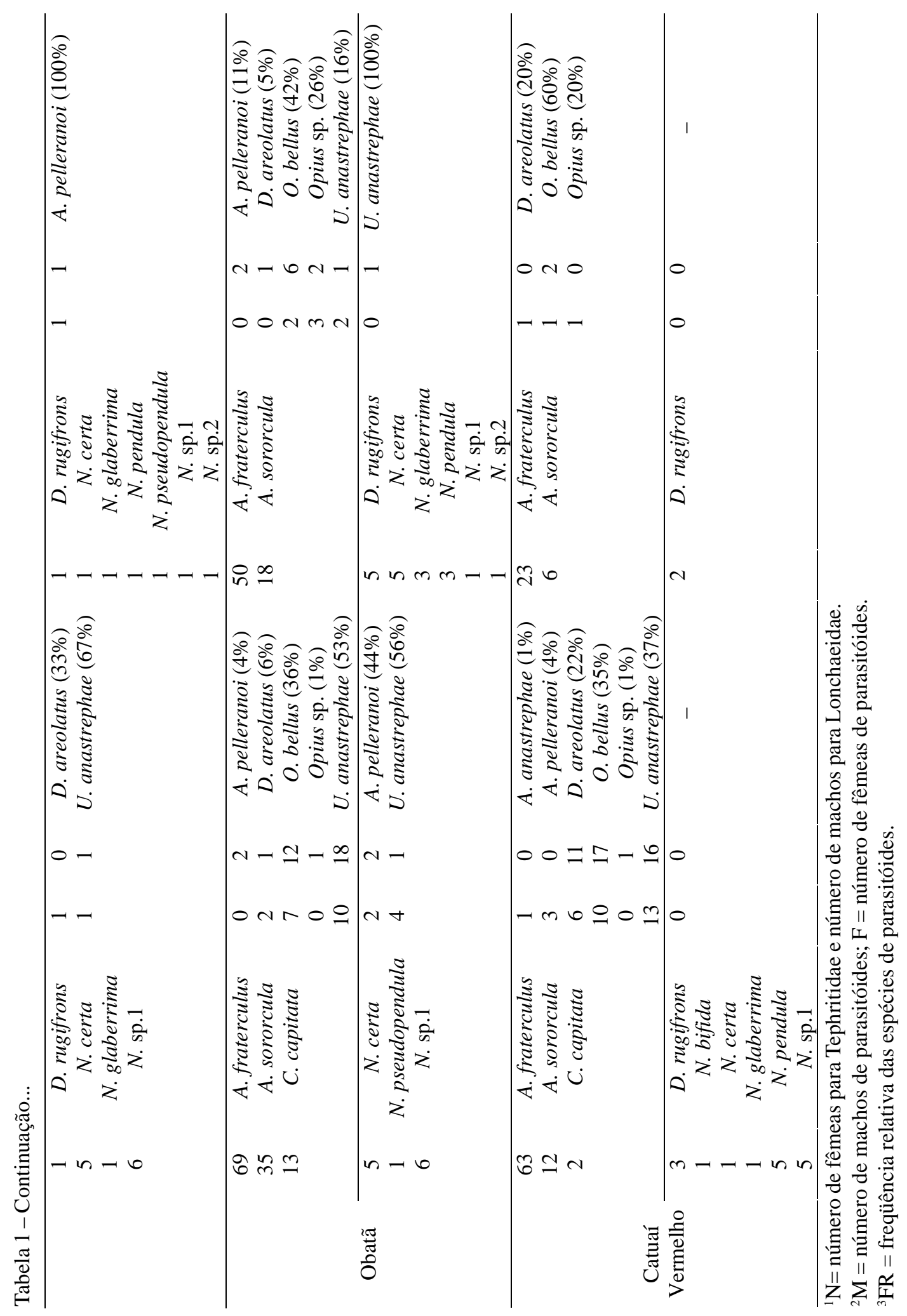

Ciênc. agrotec., Lavras, v. 32, n. 6, p. 1824-1831, nov./dez., 2008 
para cada um dos sistemas de cultivo de café. As porcentagens de parasitismo variaram de $20,4 \%$ a $12,1 \%$ e de $10,3 \%$ a $5,8 \%$ entre os seis cultivares nos sistemas sem e com arborização, respectivamente. Essas diferenças podem ser resultantes das diferenças nas características morfológicas, físicas e/ou químicas dos frutos, entre os cultivares de café, que podem influenciar o comportamento de busca e oviposição dos parasitóides (VINSON, 1976). Leonel Junior et al. (1996) observaram 7,9\% de parasitismo de moscas-das-frutas infestando café em Limeira e Piracicaba (SP) e Raga et al. (2002) registraram porcentagens de parasitismo total variando de $0,26 \%$ a $0,6 \%$. Torres (2004) obteve a mesma porcentagem de parasitismo total das larvas de tefritídeos $(4,4 \%)$ infestando Catuaí Amarelo, em sistemas arborizado e a pleno sol.

\section{CONCLUSÕES}

Os seis cultivares de café arábica avaliados são susceptíveis à infestação por moscas-das-frutas das famílias Tephritidae e Lonchaeidae, tanto no sistema de cultivo sem arborização como no arborizado. Por outro lado, espécies de Braconidae e Figitidae são capazes de parasitar larvas dessas moscas infestando os seis cultivares de café arábica, em ambos os sistemas de cultivo.

\section{REFERÊNCIAS BIBLIOGRÁFICAS}

ABASA, R. O. Observations on the seasonal emergence of fruit on a Kenya coffee estate and studies of the pest status of Ceratitis capitata Wied. in coffee. East African Agricultural and Forestry Journal, Nairobi, v. 39, n. 2, p. 144-148, 1973.

AGUIAR-MENEZES, E. L. Diversidade vegetal: uma estratégia para o manejo de pragas em sistemas sustentáveis de produção agrícola. Seropédica: Embrapa Agrobiologia, 2004. 68 p. (Documentos, 177).

AGUIAR-MENEZES, E. L.; MENEZES, E. B. Flutuação populacional das moscas-das-frutas e sua relação com a disponibilidade hospedeira em Itaguaí, RJ. Anais da Sociedade Entomolológica do Brasil, Londrina, v. 25, p. 223-232, 1996.

AGUIAR-MENEZES, E. L.; MENEZES, E. B. Rio de Janeiro. In: MALAVASI, A.; ZUCCHI, R. A. (Eds.). Moscas-das- frutas de importância econômica no Brasil: conhecimento básico e aplicado. Ribeirão Preto: Fapesp-Holos, 2000. p. 259-263.

ALUJA, M. Fruit fly (Diptera: Tephritidae) research in Latin America: myths, realities and dreams. Anais da Sociedade Entomológica do Brasil, Londrina, v. 28, n. 4, p. 565-594, 1999.

BEER, J. ¿Café bajo sombra en América Central: hace falta más investigaciones sobre este sistema agroforestal exitoso? Agroforestería en las Américas, Turrialba, v. 4, p. 8-13, 1997.

CIVIDANES, F. J.; NAKANO, O.; MELO, O. Avaliação da qualidade de frutos de café atacados por Ceratitis capitata (Wiedemann, 1824) (Diptera: Tephritidae). Scientia Agricola, Piracicaba, v. 50, n. 2, p. 220-225, 1993.

ESKAFI, F. M. Parasitism of fruit flies Ceratitis capitata and Anastrepha spp. (Diptera: Tephritidae) in Guatemala. Entomophaga, Paris, v. 35, p. 355-362, 1990.

ESKAFI, F. M.; CUNNINGHAM, R. T. Host plants of fruit flies (Diptera: Tephritidae) of economic importance in Guatemala. Florida Entomolologist, Lutz, v. 70, p. 117-123, 1987.

GONÇALVES, C. R. As moscas das frutas e seu combate. Rio de Janeiro: Ministério da Agricultura, Departamento Nacional de Produção Vegetal, Serviço de Defesa Sanitária Vegetal, 1938. 43 p. (Folheto, 12).

GUIMARÃES, J. A.; DIAZ, N. B.; ZUCCHI, R. A. Parasitóides - Figitidae (Eucoilinae). In: MALAVASI, A.; ZUCCHI, R. A. (Eds.). Moscas-das-frutas de importância econômica do Brasil: conhecimento básico e aplicado. Ribeirão Preto: Fapesp-Holos, 2000. p. 127-135.

GUIMARÃES, J. A.; ZUCCHI, R. A.; DIAZ, N. B.; SOUZA FILHO, M. F.; UCHOA, F. M. A. Espécies de Eucoilinae (Hymenoptera: Cynipoidea: Figitidae) parasitóides de larvas frugívoras (Diptera: Tephritidae e Lonchaeidae) no Brasil. Anais da Sociedade Entomologia do Brasil, Londrina, v. 28, p. 263-273, 1999. 
HAGGAR, J.; STAVER, C.; MELO, E. Sostenibilidad y sinergismo en sistemas agroforestales con café: estudio de interacciones entre plagas, fertilidad del suelo y árboles de sombra. Agroforestería en las Américas, Turrialba, v. 8, n. 29, p. 22-29, 2001.

KATIYAR, K. P.; CAMACHO, M. J.; MATHEU, R. Parasitóides hymenópteros de moscas de las frutas (Diptera: Tephritidae) en la región occidental de Venezuela. Revista de la Facultad de Agronomía (LUZ), Maracaibo, v. 12, p. 303-312, 1995.

LEONEL JUNIOR, F. L.; ZUCCHI, R. A.; CANAL, D. N. A. Parasitismo de moscas-das-frutas (Diptera: Tephritidae) por Braconidae (Hymenoptera) em duas localidades do estado de São Paulo. Anais da Sociedade Entomologia do Brasil, Londrina, v. 25, p. 199-206, 1996.

LÓPEZ, M.; ALUJA, M.; SIVINSKI, J. Hymenopterous larval-pupal and pupal parasitoids of Anastrepha flies (Diptera: Tephritidae) in Mexico. Biological Control, Orlando, v. 15, p. 119-129, 1999.

MALAVASI, A.; ZUCCHI, R. A. Moscas-das-frutas de importância econômica do Brasil: conhecimento básico e aplicado. Ribeirão Preto: Fapesp-Holos, 2000. 327 p.

MATIELLO, J. B.; SIQUEIRA, H. V. A. Café no estado do Rio de Janeiro. Rio de Janeiro: FAERJ/SEBRAE-RJ, 1999. $51 \mathrm{p}$.

MCALPINE, J. F.; STEYSKAL, G. C. A revision of Neosilba McAlpine with a key to the world genera of Lonchaeidae (Diptera). Canadian Entomologist, Ottawa, v. 114, p. 105137, 1982.

NEVES, A. M. B.; TOFANI, S. R. M.; GERALDO, B. S.; SILVA, E. R. Mosca no café. Cultivar, Pelotas, v. 4, p. 3435, 2002.

RAGA, A.; PRESTES, D. A. O.; SOUZA FILHO, M. F.; SATO, M. E.; SILOTO, R. C.; ZUCCHI, R. A. Occurrence of fruit flies in coffee varieties in the state of São Paulo, Brazil. Boletín de Sanidad Vegetal y Plagas, v. 28, p. 519-524, 2002.

RAGA, A.; SOUZA FILHO, M. F.; ARTHUR, V.; MARTINS, A. L. M. Avaliação da infestação de moscas- das-frutas em variedades de café (Coffea spp.). Arquivos do Instituto Biológico, São Paulo, v. 63, p. 59-63, 1996.

RICCI, M. S. F.; NEVES, M. C. P. (Eds.). Cultivo orgânico de café. Seropédica: Embrapa Agrobiologia, 2006. (Sistema de Produção, 2). Disponível em: <http:// Www.cnpab.embrapa.br/pubblicacoes/sistemasdeproducaool tcafe/index.htm' > Acesso em: 19 nov. 2007.

SIVINSKI, J.; ALUJA, M.; HOLLER, T. The distributions of the Caribbean fruit fly, Anastrepha suspensa (Tephritidae) and its parasitoids (Hymenoptera: Braconidae) within the canopies of host trees. Florida Entomologist, Lutz, v. 82, n. 1, p. 72-81, 1999.

SPOLIDORO, M. L. C. V. Composição e estrutura de um trecho de floresta no Médio Paraíba do Sul, RJ. 2001. 90 p. Dissertação (Mestrado em Ciências Ambientais) Universidade Federal Rural do Rio de Janeiro, Seropédica, 2001.

TORRES, C. A. S. Diversidade de espécies de moscasdas-frutas (Diptera: Tephritidae) e de seus parasitóides em cafeeiro (Coffea arabica L.). 2004. 71 p. Dissertação (Mestrado em Agronomia) Universidade Estadual do Sudoeste da Bahia, Vitória da Conquista, 2004.

VINSON, S. B. Host selection by insect parasitoids. Annual Review of Entomology, Palo Alto, v. 21, p. 109-133, 1976.

WHARTON, R. A.; GILSTRAP, F. E.; RHODE, R. H.; FISCHEL, M.; HART, W. G. Hymenopterous egg-pupal and larval-pupal parasitoids of Ceratitis capitata and Anastrepha spp. (Dip.: Tephritidae) in Costa Rica. Entomophaga, Paris, v. 26, p. 285-290, 1981.

WHARTON, R. A.; MARSH, P. M. New world opiinae (Hymenoptera: Braconidae) parasitic on Tephritidae (Diptera). Journal of Washington Academic Science, Washington, v. 68, p. 147-167, 1978.

WHITE, I. M.; ELSON-HARRIS, M. M. Fruit flies of economic significance: their identification and bionomics. Wallingford: CAB International, 1992. 601 p. 
YEPES, R. F.; VELEZ, A. R. Contribución al conocimiento de las moscas de las frutas (Tephritidae) y sus parasitóides en el departamento de Antioquia. Revista de la Facultad Nacional de Agricultura, Medellín, v. 42, p. 73-98, 1989.
ZUCCHI, R. A. Taxonomia. In: MALAVASI, A.; ZUCCHI, R. A. (Eds.). Moscas-das-frutas de importância econômica do Brasil: conhecimento básico e aplicado. Ribeirão Preto: Fapesp-Holos, 2000. p. 13-24. 